\title{
Perfiles motivacionales a metas y manejo de estrategias de afrontamiento de estrés en estudiantes universitarios
}

\author{
Victoria Franco Taboada, Ramón González Cabanach, y Antonio Souto Gestal \\ Universidad de La Coruña (España)
}

\begin{abstract}
Se diseñó una investigación para conocer la posible vinculación entre las orientaciones motivacionales de los estudiantes y la elección de unas u otras estrategias de afrontamiento ante las experiencias de estrés académico, y comprobar, asimismo, si éstas podrían variar en función de las distintas orientaciones a metas. Los participantes fueron 468 estudiantes universitarios con una media de edad de 21,82 años $(D T=3,13)$ que un análisis quick cluster agrupó en 5 conglomerados con combinaciones diferentes de orientaciones a metas de aproximación y evitación al aprendizaje y al rendimiento. El factor de afrontamiento que concitó mayor unanimidad en las respuestas en todas las agrupaciones de orientaciones a metas fue Búsqueda de apoyo social. No fue este el caso de los otros dos factores, Reevaluación positiva y Planificación y gestión de recursos, con una presencia mucho más dispar entre unos grupos y otros según sus orientaciones motivacionales, al mostrarse diferencias significativas en su manejo entre las agrupaciones de evitación y aproximación a favor de estas últimas. Sería conveniente proporcionar a los universitarios programas para aprender a manejar el estrés, fomentar los recursos de afrontamiento, promover el apoyo motivacional y la afectividad positiva, y formar habilidades para saber responder a las demandas académicas y evitar su vulnerabilidad ante el estrés que generan.
\end{abstract}

Palabras clave: Metas, aproximación, evitación, afrontamiento, estrés.

Motivational profiles to coping stress strategies goals and management in university students: This research was designed to know the possible correlation among the motivational orientations of the students and the choices of the coping strategies they make before the different experiences of academic stress, and, additionally, to know if this could change based on the different orientation to goals. The participants were 468 university students with an average age of 21.82 years old $(D T=3.13)$. A quick cluster analysis classified them into 5 groups with different orientations of approximation goals, and learning and performance avoidance. The coping factor that caused a greater degree of unanimity in the responses of all the orientation groups was Search for Social Support. This was not the case of the other two factors, Positive Reassessment and Resource Planning and Management, with a much more uneven presence among some groups and others, according to their motivational orientations, as there were significant differences between the avoidance and approximation groups in favour of the latter. It would be convenient to provide programmes to the students in order to learn to manage stress, to encourage the coping resources, to promote the motivational support and the positive affectivity, and to train abilities in order to know how to respond to the academic demands, and so, avoiding their vulnerability to the stress they generate

Keywords: Goals, approximation, avoidance, coping, stress. 
El concepto de afrontamiento alude al esfuerzo cognitivo y conductual activado por el individuo ante exigencias situacionales externas o internas, apreciadas como excesivas para los recursos de los que dispone (Lazarus y Folkman, 1986). Su objetivo es mitigar los efectos negativos sobre el propio estado de bienestar, según la valoración previa del acontecimiento estresante. Se trata de una evaluación de carácter claramente subjetivo, porque el sujeto valora la gravedad del suceso sopesando sus experiencias previas, el momento particular en que se produce y los recursos de los que dispone (Fernández y Díaz, 2001; Richardson y Poole, 2001).

Ante cualquier situación de estrés que conlleve una percepción de amenaza o desafío, partimos de que las decisiones de actuación son consecuencia de la valoración que sustenta esta percepción y sobre la que se construye la necesidad de actuar o no, y de las medidas cognitivas, conductuales y emocionales que se elegirán para encararla con el menor riesgo posible para el equilibrio y el bienestar personal. Esta percepción subjetiva explicaría las divergencias interindividuales de conducta ante el mismo estímulo, así como la toma de decisiones particulares para hacerle frente, ya sea recurriendo a un afrontamiento dirigido a la resolución del problema porque el individuo estima que el suceso es modificable y que puede controlarlo, u optando por un afrontamiento dirigido a la emoción al apreciar el sujeto la inmodificabilidad del suceso y orientar su actuación a mitigar sus consecuencias (Lazarus y Folkman, 1986; Skinner, Edge, Altman, y Sherwood, 2003).

Ya desde la primera evaluación, los componentes motivacionales ocupan un lugar central en la misma, bien por su relevancia o bien por su congruencia o incongruencia. Según Fernández-Abascal (1999), la relevancia motivacional se relaciona con los compromisos de la persona y en qué nivel la situación puede afectarles, mientras que la congruencia motivacional evalúa el grado de consistencia con las aspiraciones y metas que el sujeto mantiene. Por lo tanto, en todo el proceso de valoración, ocupan un lugar preponderante los compromisos o metas de la persona, los cuales podemos considerarlos como las obligaciones contraídas por el propio individuo con respecto a sí mismo y a su entorno inmediato: "expresan lo que es importante para el individuo y determinan sus decisiones" (Lazarus y Folkman, 1986, p. 103), razón por la que las metas a las que aspira tendrán una influencia directa en las consecuencias de las decisiones de afrontamiento que se pongan en marcha, para que no interfieran con las mismas o, en todo caso, ayuden al sujeto a alcanzarlas.

\section{Objetivos}

En nuestro estudio queremos analizar cómo afrontan los alumnos las situaciones asociadas al estrés académico y las relaciones entre los tipos de metas académicas que los motivan y su posible incidencia diferencial en la elección de estrategias para manejar el estrés. Son escasísimos los trabajos que vinculan las metas de 
dominio y las metas de rendimiento con formas específicas de afrontamiento. Además, se han encontrado resultados contradictorios con respecto a las motivaciones, en su vertiente de evitación y de aproximación, y la experiencia emocional asociada, aspecto que también intentaremos clarificar en la presente investigación.

\section{MÉTODO}

\section{Descripción de la muestra}

La muestra utilizada en el estudio estuvo formada por 468 estudiantes de diferentes ramas de conocimiento (Titulaciones Técnicas, 44.9\%; Ciencias Sociales y Jurídicas, 12.8\%; Ciencias de la Educación, 31.2\%, y Ciencias de la Salud, 11.1\%) de la Universidad de A Coruña. El muestreo ha sido por conglomerados o grupos establecidos de estudiantes por clase, con una participación voluntaria (muestra aceptante) en la recogida de datos por medio de cuestionarios. La distribución de la muestra fue de 292 mujeres (62.4\%) y 176 hombres (37.6\%) entre 18 y 44 años con una media de edad de 21.82 años $(D T=3,13)$.

\section{Instrumentos de medida}

Escala de Afrontamiento del Estrés (A-CEA). La escala A-CEA de Cabanach, Valle, Rodríguez y Piñeiro (2008) contiene 23 ítems que evalúan las estrategias cognitivas y conductuales a las que recurre el estudiante para hacer frente a las situaciones y/o circunstancias generadoras de estrés académico. Es una escala con respuestas tipo Likert a cada ítem con cinco opciones: Nunca (1), Alguna vez (2), Bastantes veces (3), Muchas veces (4) y Siempre (5). Presenta una estructura factorial de tres dimensiones que pueden explicar el $61.554 \%$ de la varianza total y que se distribuyen en reevaluación positiva, búsqueda de apoyo social, y planificación y gestión de recursos personales.

La aplicación de esta escala en otras investigaciones (Cabanach, Valle, Rodríguez, Piñeiro, y Freire, 2010) mostró unos valores de consistencia interna muy buenos, con un coeficiente de Cronbach general de la escala de ,890. En este estudio la consistencia interna muestra un valor para el global de la escala igual a, 909, muy cerca del valor máximo de fiabilidad (1).

Escala de Orientación a Metas. Esta escala (Skaalvik, 1997) está formada por 21 ítems y mide la existencia de dos orientaciones distintas (aproximación y evitación) en las metas de aprendizaje y de rendimiento. La respuesta a cada ítem se basa en una escala de cinco puntos en la que el estudiante puede elegir entre distintas opciones: Nunca (1), Casi nunca (2), Algunas veces (3), Casi siempre (4) y Siempre (5).

Se evalúan cuatro factores que conforman diferentes orientaciones de motivación en los estudiantes: metas de aproximación al aprendizaje, metas de evitación 
del aprendizaje, metas de aproximación al rendimiento y metas de evitación del rendimiento. El análisis factorial exploratorio informa de una estructura de cuatro dimensiones que pueden explicar el $53.33 \%$ de la varianza total. Con respecto a la fiabilidad, obtenida mediante el alpha de Cronbach, osciló desde .696 y .870 para cada uno de los cuatro factores considerados y mostró un valor $\alpha$ para el global de la escala igual a .749 .

\section{Procedimiento de recogida de información y análisis de datos}

Se diseñó un plan de investigación de naturaleza no experimental y de carácter transversal, obteniendo la información de los estudiantes de la muestra mediante los cuestionarios ya descritos y a los que cada grupo por curso establecido contestó durante el horario normal de clase de forma voluntaria y anónima. Previamente se les explicó de forma sucinta lo que se iba a investigar, la voluntariedad de su participación y su anonimato si así lo deseaban. Se informó de la completa confidencialidad de los datos y de la nula relación entre los resultados de los cuestionarios y su expediente académico, por lo que se les pedía sinceridad en sus respuestas.

Dado el carácter descriptivo y comparativo del diseño de investigación por el que hemos optado en este estudio, no podemos hablar de variables independientes propiamente dichas, porque no se produce una manipulación directa durante la investigación, aunque sí podemos considerar como variables independientes asignadas las variables motivacionales utilizadas para conformar agrupaciones de individuos de acuerdo con unos valores determinados y poder, así, observar si se producen diferencias entre ellas con respecto a las variables dependientes, representadas por las estrategias de afrontamiento que activan los estudiantes para manejar las situaciones de estrés académico. El análisis de resultados se llevó a cabo con el programa SPSS versión 22.0 para Windows (IBM Corp., 2013).

Inicialmente se investigaron las estrategias de afrontamiento y las orientaciones a metas de los estudiantes mediante análisis descriptivos de las puntuaciones medias obtenidas y, después, se comprobó qué vinculaciones se producían entre las orientaciones motivacionales y el manejo de estrategias de afrontamiento realizando un cálculo de correlaciones bivariadas (Pearson).

Con el propósito de identificar diferentes grupos de orientaciones motivacionales sin especificar a priori las agrupaciones a formar, para el análisis clúster se ha utilizado un método no jerárquico como el "quick cluster analysis" con el fin de optimizar el criterio de selección y que permite reasignar un individuo, previamente asignado a un grupo en un paso precedente, a otro en un paso posterior (Bisquerra, 1989). Una vez establecidas las diferentes agrupaciones se estudió la diferencia de medias de las variables de afrontamiento de estrés con el análisis de varianza ANOVA y 
se empleó Scheffé como prueba de contraste post hoc para identificar en qué agrupaciones en particular resultaban significativas las posibles diferencias.

\section{RESULTADOS}

Análisis de la relación entre estrategias de afrontamiento y orientaciones a metas

Como primer paso se analizaron las relaciones entre las estrategias de afrontamiento de estrés y las diferentes orientaciones a metas académicas de los estudiantes de la muestra (metas de aproximación y evitación del aprendizaje, y metas de aproximación y evitación del rendimiento), partiendo de los índices de correlación entre las variables dependientes e independientes (ver Tabla 1).

Los estudiantes con metas de evitación del rendimiento o "metas de defensa del yo" no establecen ninguna correlación con Búsqueda de Apoyo social y sí relaciones inversas y negativas con Reevaluación positiva y Planificación, lo que nos informa de que cuanto más se preocupa el estudiante por no presentar una mala imagen de sí mismo ante los demás, menos recurre a manejar estrategias de afrontamiento activo, cognitivo y conductual para afrontar sus dificultades académicas.

Por el contrario, los estudiantes con metas de aproximación al rendimiento o "metas de mejora del yo" establecen correlaciones directas y positivas, aunque bajas, tanto con Reevaluación positiva como con Planificación, pero en el caso de Planificación a un nivel de significación de 0.01 de la variable frente a Reevaluación positiva a un nivel de significación de 0.05. Con Búsqueda de apoyo, sin embargo, mantienen una relación inversa y negativa, aunque baja y bastante por debajo del 0.30 de magnitud, y a un nivel de significación del 0,05.

Por su parte, los datos de los estudiantes con metas de aproximación al aprendizaje o "metas de dominio o maestría" no presentan correlación con Reevaluación positiva, pero sí de forma positiva y directa con Búsqueda de apoyo y con Planificación, aunque en magnitudes bastante bajas, muy por debajo del .30, presentando, sin embargo, en Planificación un nivel de significación de 0.01 y en Búsqueda de apoyo de 0.05.

El último grupo analizado, el de los estudiantes con metas de evitación del aprendizaje, presenta correlaciones inversas y negativas con valores bajos, con los factores Planificación y Búsqueda de apoyo a un nivel alto de significación del 0.01 en la primera y de 0.05 en la segunda variable, y ninguna correlación con Reevaluación. 
FRANCO ET AL. Orientaciones a metas y afrontamiento de estrés en universitarios.

Tabla 1. Correlaciones de las diferentes orientaciones a metas académicas con las variables de estrategias de afrontamiento de estrés académico para la muestra

\begin{tabular}{|c|c|c|c|c|c|c|}
\hline \multicolumn{7}{|c|}{ CORRELACIONES } \\
\hline & & & $\begin{array}{c}\text { Ev. } \\
\text { Rend }\end{array}$ & $\begin{array}{l}\text { Aprox. } \\
\text { Rend }\end{array}$ & $\begin{array}{l}\text { Aprox. } \\
\text { Apr }\end{array}$ & $\begin{array}{l}\text { Ev. } \\
\text { Apr }\end{array}$ \\
\hline \multirow{9}{*}{$\begin{array}{l}\text { ESTRATEGIAS DE } \\
\text { AFRONTAMIENTO }\end{array}$} & \multirow{3}{*}{$\begin{array}{l}\text { REEVALUACION } \\
\text { POSITIVA }\end{array}$} & $\begin{array}{l}\text { Correlación de } \\
\text { Pearson }\end{array}$ & $-.238^{* *}$ & $.103^{*}$ & .037 & -.056 \\
\hline & & Sig. (bilateral) & .000 & .027 & .421 & .229 \\
\hline & & $\mathrm{N}$ & 462 & 462 & 462 & 462 \\
\hline & \multirow{3}{*}{$\begin{array}{l}\text { BUSQUEDA DE } \\
\text { APOYO }\end{array}$} & $\begin{array}{l}\text { Correlación de } \\
\text { Pearson }\end{array}$ & -.033 & $-.091^{*}$ & $.118^{*}$ & $-.092^{*}$ \\
\hline & & Sig. (bilateral) & .484 & .049 & .011 & .049 \\
\hline & & $\mathrm{N}$ & 462 & 462 & 462 & 462 \\
\hline & \multirow{3}{*}{ PLANIFICIACIÓN } & $\begin{array}{l}\text { Correlación de } \\
\text { Pearson }\end{array}$ & $-.114^{*}$ & $.185^{* *}$ & $.197^{* *}$ & $-.188^{* *}$ \\
\hline & & Sig. (bilateral) & .015 & .000 & .000 & .000 \\
\hline & & $\mathrm{N}$ & 462 & 462 & 462 & 462 \\
\hline
\end{tabular}

** La correlación es significativa en el nivel 0.01 (2 colas).

* La correlación es significativa en el nivel 0.05 ( 2 colas).

\section{Perfiles de orientación de metas académicas}

Buscando una vinculación más consistente entre los tipos de orientaciones a metas y las variables de afrontamiento de estrés, se realizó un análisis de conglomerados para identificar perfiles posibles de diferentes tendencias a metas $\mathrm{y}$, posteriormente, se llevó a cabo un análisis de varianza (ANOVA) para investigar las diferencias en las variables de afrontamiento en función de las agrupaciones de estudiantes. Tras estandarizar las variables del cuestionario de metas para el análisis clúster, se agruparon los estudiantes en cinco clústeres y se establecieron los perfiles de agrupamiento de acuerdo con la frecuencia de aparición de las distintas opciones motivacionales.

Se obtuvieron cinco agrupaciones de orientaciones motivacionales en las que dos grupos mostraron una única motivación a metas: los grupos de aproximación y de evitación del aprendizaje (el grupo AP_AP con 98 alumnos y un 21\% de la distribución, y el grupo EV_AP con 51 alumnos y el 11\%). Los otros tres grupos exhibieron en mayor o menor grado orientaciones a varias metas, como el grupo de metas de evitación del rendimiento (EV_RD con 98 alumnos y un 21\% de la distribución) que manifestó alguna orientación a metas de aproximación al aprendizaje y al rendimiento; el grupo de metas de aproximación al rendimiento (AP_RD con 104 alumnos y un 22\% de la distribución) que se inclinó en ciertas tareas por una orientación de aproximación al aprendizaje, y, por último, un grupo que combinó metas de evitación del aprendizaje y metas de evitación del rendimiento (EV_AP+EV_RD con 117 alumnos y un 25\% de la distribución) con preferencia por las del primer tipo. 
FRANCO ET AL. Orientaciones a metas y afrontamiento de estrés en universitarios.

Tabla 2. Distribución de estudiantes por grupo según tipo de metas motivacionales

\begin{tabular}{lccc}
\hline CLÚSTER & $\begin{array}{c}\mathrm{N}^{\circ} \text { DE SUJETOS } \\
\text { /GRUPO }\end{array}$ & PORCENTAJE & DESCRIPCIÓN GRUPO \\
\hline 1 (AP_AP) & 98,000 & $21 \%$ & $\begin{array}{c}\text { Grupo de Aproximación al } \\
\text { Aprendizaje }\end{array}$ \\
\hline 2 (EV_RD) & 98,000 & $21 \%$ & $\begin{array}{c}\text { Grupo de Evitación del } \\
\text { Rendimiento }\end{array}$ \\
\hline $3($ EV_AP) & 51,000 & $11 \%$ & $\begin{array}{c}\text { Grupo de Evitación del } \\
\text { Aprendizaje }\end{array}$ \\
\hline $4($ EV_AP + & 117,000 & $25 \%$ & $\begin{array}{c}\text { Grupo de Evitación del } \\
\text { Apendizaje y de Evitación } \\
\text { del Rendimiento }\end{array}$ \\
\hline EV_RD) & 104,000 & $22 \%$ & $\begin{array}{c}\text { Grupo de Aproximación al } \\
\text { Rendimiento }\end{array}$ \\
\hline 5 (AP_RD) & 468,000 & $100 \%$ & \\
\hline VÁLIDO &, 000 & & \\
\hline PERDIDOS & &
\end{tabular}

Figura 1. Representación gráfica de clústeres finales. Grupos de estudiantes por tipo de metas

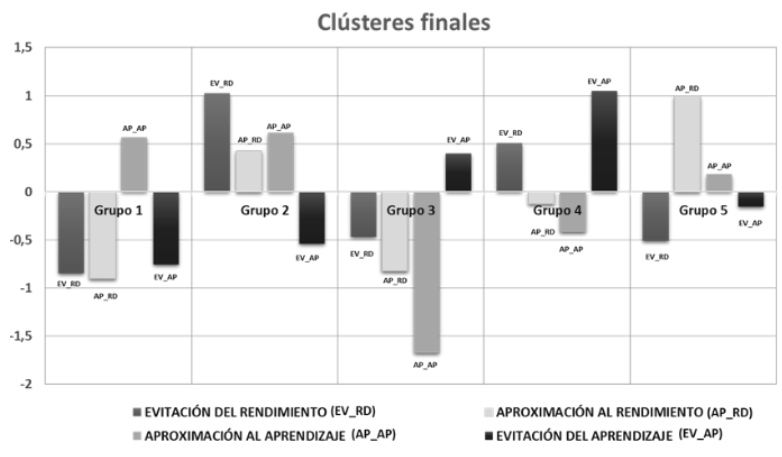

Afrontamiento por grupos de tendencias a metas

Para estudiar las opciones estratégicas de afrontamiento elegidas por las distintas agrupaciones se ha recurrido a la escala A_CEA con tres factores: Reevaluación positiva, Búsqueda de apoyo y Planificación y gestión de recursos, cuyos descriptivos de los valores de las medias y de las desviaciones típicas para cada grupo se reflejan en la tabla 3 .

La Búsqueda de apoyo social y emocional es el tipo de afrontamiento al que más recurren los distintos grupos de estudiantes, con independencia de su orientación a metas. Se trata de una forma de afrontamiento conductual que busca apoyo, información y consejo de otros para afrontar las dificultades académicas que se presentan. Las otras dos estrategias son de carácter netamente cognitivo y activo, y se dirigen claramente a la solución de problemas. La reevaluación positiva reinterpreta la situación para verla desde una perspectiva menos negativa, analizándola en sus aspectos más favorables. Con la Planificación se efectúa un análisis de la propia situación para desarrollar un plan de acción y poder modificar la relación con un entorno generador de estrés. 
FRANCO ET AL. Orientaciones a metas y afrontamiento de estrés en universitarios.

Tabla 3. Medias y desviaciones típicas de los grupos de estudiantes con diferentes metas en relación a las estrategias de afrontamiento

\begin{tabular}{|c|c|c|c|c|c|c|c|c|c|}
\hline \multicolumn{10}{|c|}{ DESCRIPTIVOS } \\
\hline \multirow[b]{2}{*}{$\begin{array}{l}\text { Estrategias } \\
\text { Afrontamiento }\end{array}$} & \multirow[b]{2}{*}{ Grupos } & \multirow[b]{2}{*}{$\mathrm{N}$} & \multirow[b]{2}{*}{ Media } & \multirow[b]{2}{*}{$\begin{array}{l}\text { Desviación } \\
\text { estándar }\end{array}$} & \multirow[b]{2}{*}{$\begin{array}{l}\text { Error } \\
\text { estándar }\end{array}$} & \multicolumn{2}{|c|}{$\begin{array}{c}95 \% \text { del intervalo de } \\
\text { confianza para la } \\
\text { media }\end{array}$} & \multirow[b]{2}{*}{ Mínimo } & \multirow[b]{2}{*}{ Máximo } \\
\hline & & & & & & $\begin{array}{l}\text { Límite } \\
\text { inferior }\end{array}$ & $\begin{array}{c}\text { Límite } \\
\text { superior }\end{array}$ & & \\
\hline \multirow{6}{*}{$\begin{array}{l}\text { Reevaluación } \\
\text { Positiva }\end{array}$} & $1 \mathrm{AP} \_\mathrm{AP}$ & 96 & 2.9363 & .84600 & .08634 & 2.7649 & 3.1078 & 1.11 & 4.56 \\
\hline & $2 \mathrm{EV} \_\mathrm{RD}$ & 96 & 2.6369 & .80718 & .08238 & 2.4733 & 2.8004 & 1.00 & 4.78 \\
\hline & 3 EV_AP & 51 & 2.8324 & .92229 & .12915 & 2.5730 & 3.0918 & 1.33 & 4.56 \\
\hline & $\begin{array}{c}4 \\
\mathrm{EV} \_\mathrm{AP}+\mathrm{EV} \\
\text { _RD }\end{array}$ & 115 & 2.6348 & .71461 & .06664 & 2.5028 & 2.7668 & 1.00 & 4.67 \\
\hline & 5 AP_RD & 104 & 3.0791 & .84643 & .08300 & 2.9144 & 3.2437 & 1.22 & 4.78 \\
\hline & Total & 462 & 2.8197 & .83300 & .03875 & 2.7435 & 2.8959 & 1.00 & 4.78 \\
\hline \multirow{11}{*}{$\begin{array}{l}\text { Búsqueda } \\
\text { de Apoyo }\end{array}$} & $1 \mathrm{AP} \_\mathrm{AP}$ & 96 & 3.4286 & .96624 & .09862 & 3.2328 & 3.6243 & 1.57 & 5.00 \\
\hline & 2EV_RD & 96 & 3.3839 & 1.07990 & .11022 & 3.1651 & 3.6027 & 1.14 & 5.00 \\
\hline & 3 EV_AP & 51 & 3.1214 & 1.10330 & .15449 & 2.8111 & 3.4317 & 1.29 & 5.00 \\
\hline & $\begin{array}{c}4 \\
\mathrm{EV} \_\mathrm{AP}+\mathrm{EV} \\
\text { _RD } \\
\end{array}$ & 115 & 3.2280 & .95501 & .08906 & 3.0515 & 3.4044 & 1.00 & 5.00 \\
\hline & 5 AP_RD & 104 & 3.1690 & .96291 & .09442 & 2.9817 & 3.3562 & 1.00 & 5.00 \\
\hline & Total & 462 & 3.2770 & 1.00524 & .04677 & 3.1851 & 3.3689 & 1.00 & 5.00 \\
\hline & 2 EV_RD & 96 & 2.8929 & .80812 & .08248 & 2.7291 & 3.0566 & 1.43 & 4.71 \\
\hline & 3 EV_AP & 51 & 2.3805 & .80717 & .11303 & 2.1535 & 2.6075 & 1.14 & 4.29 \\
\hline & $\begin{array}{c}4 \\
\mathrm{EV} \_\mathrm{AP}+\mathrm{EV} \\
\text { _RD }\end{array}$ & 115 & 2.6234 & .75646 & .07054 & 2.4837 & 2.7631 & 1.14 & 4.57 \\
\hline & 5 AP_RD & 104 & 3.0714 & .83655 & .08203 & 2.9087 & 3.2341 & 1.00 & 5.00 \\
\hline & Total & 462 & 2.8246 & .84270 & .03921 & 2.7475 & 2.9016 & 1.00 & 5.00 \\
\hline
\end{tabular}

El grupo con un valor de media más alto en Búsqueda de apoyo $(M=3.42)$ es el de metas AP_AP. También alcanza medias bastante altas en Reevaluación $(M=2.93)$ y Planificación y gestión de recursos $(M=2.96)$. Este grupo se caracteriza por el deseo de aprender, orientando su trabajo académico a mejorar su capacitación, así que es lógico que combine los tres tipos de estrategias para hacer frente a las dificultades que se les presenten durante el desarrollo de las actividades académicas.

El grupo AP_RD presenta la media más elevada en Reevaluación $(M=3.07)$ y Planificación $(M=3.07)$. Su principal motivación es hacia la mejora del yo y lograr un mejor rendimiento que los demás para demostrar que, comparativamente, disfrutan de una capacidad superior; pero no debemos olvidar que esta agrupación combina, aunque en un porcentaje mucho menor y sólo ante determinadas tareas, metas de aproximación al aprendizaje. El recurso a estas estrategias cognitivas, activas y positivas presenta a estos estudiantes como un grupo muy bien adaptado a las exigencias propias de la actividad académica, aun recurriendo en menor medida que el resto de los grupos a la 
Búsqueda de apoyo $(M=3.16)$, tal vez porque puedan considerarla, en su vertiente emocional, una muestra de debilidad o porque se consideran autosuficientes para resolver sus propios problemas sin ayudas externas.

El clúster con las medias más bajas en los factores Búsqueda de apoyo $(M=3.12)$ y Planificación $(M=2.38)$ es el EV_AP. Esta agrupación de estudiantes está claramente definida en su tendencia a evitar el esfuerzo y el trabajo académico, así que no es raro que recurra más a menudo a la búsqueda de un Apoyo social que a la Planificación para afrontar las dificultades académicas, estrategia que está lejos de utilizar a menudo, aunque en la Reevaluación obtiene una media no muy baja $(M=2.83)$.

Los grupos de metas EV_RD y de metas EV_AP+EV_RD se inclinan claramente por Búsqueda de apoyo $(M=3.38)$ y $(M=3.22)$ respectivamente, frente a la media más baja en ambos casos en Reevaluación $(M=2.63)$. Estos clústeres agrupan estudiantes con una clara motivación para proteger su propia imagen y eludir valoraciones negativas de sí mismos por parte de otras personas. Tal vez el manejo de una estrategia activa y positiva como la Reevaluación no es compatible con un tipo de motivación negativa tan marcada hacia la actividad académica. Además, el grupo EV_AP+EV_RD presenta una tendencia a rehuir el esfuerzo y las obligaciones académicas, lo que podría explicar su baja media en Planificación $(M=2.62)$.

\section{DISCUSIÓN Y CONCLUSIONES}

En la vida universitaria los estudiantes se encuentran inmersos en múltiples situaciones estresantes en relación a diferentes aspectos de la experiencia académica, por lo que es especialmente interesante analizar cómo evalúan y cómo se enfrentan a ellas, a qué tipo de recursos y estrategias recurren para afrontarlas y en qué circunstancias.

El concepto de afrontamiento del modelo transaccional de estrés, derivado de la percepción subjetiva del individuo, es considerado como un proceso dinámico de elección de acciones que puede cambiar de acuerdo con la situación y según las modificaciones que en la misma se produzcan, por lo que se han llevado a cabo trabajos para investigar la dinámica interactiva entre las percepciones particulares del sujeto, sus intenciones, expectativas o metas, y la elección de las estrategias de afrontamiento ante determinados acontecimientos estresores. Así, ante un evento potencialmente estresante que interfiera con algún compromiso personal previo, será inevitable que este último influya en la valoración que realice el sujeto del suceso, considerando que las consecuencias de las decisiones de afrontamiento que se adopten no conlleven resultados negativos para el compromiso previamente establecido o que, si ello es posible, faciliten su cumplimiento. Investigar esta cuestión fue el objetivo principal del estudio que presentamos: averiguar en qué medida los compromisos académicos o metas de nuestros 
estudiantes influyen o determinan la elección de unas u otras estrategias de afrontamiento de estrés académico en sus estudios universitarios.

La herramienta utilizada para la recogida de la información acerca de las estrategias de afrontamiento que manejan los estudiantes sólo contempla estrategias activas, principalmente de carácter cognitivo y conductual. La puntuación media obtenida para los tres factores de la escala es de 2.93 sobre 5 , un manejo bastante óptimo de este tipo de estrategias, orientadas claramente a la resolución de problemas. El factor de afrontamiento que concita mayor unanimidad en las respuestas en todas las agrupaciones de orientaciones a metas es Búsqueda de apoyo $(M=3.27)$. Los otros dos factores, Reevaluación positiva y Planificación de recursos, muestran idéntico resultado $(M=2.8)$, pero con una presencia mucho más dispar entre unos grupos y otros según sus orientaciones a metas.

Es importante valorar que las estrategias de Búsqueda de apoyo pueden funcionar como un buen protector contra el estrés. Como conjunto de estrategias ofrece una doble dimensión de la ayuda de tipo conductual (obtener consejo e información) y emocional (buscar comprensión y alivio emocional). Ambas orientaciones son adaptativas y eficientes a la hora de afrontar las dificultades académicas y reducir sus consecuencias. Según nuestros resultados, los estudiantes, con independencia de su orientación motivacional a metas, se inclinan de forma preferente por hablar con personas emocionalmente cercanas, por pedir consejo a alguien al que respeten y por obtener más información sobre la situación, lo que podríamos considerar como una búsqueda de apoyo de carácter informativo y de asesoramiento. Según Cabanach, Fariña, Freire, González, y Ferradas (2013), este tipo de actuación debe diferenciarse del establecimiento de una dependencia de los otros para solventar los problemas, lo que limitaría la eficacia de la estrategia.

Con respecto a los otros dos factores de afrontamiento medidos, Reevaluación positiva y Planificación y gestión de recursos, que son estrategias cognitivas y activas dirigidas principalmente a la solución de problemas, sí se observan disparidades significativas en su manejo entre los grupos de evitación y aproximación a favor de estos últimos. Los dos grupos de metas de aproximación al rendimiento (AP_RD) y de aproximación al aprendizaje (AP_AP) son los que más las utilizan, y los tres grupos de evitación los que menos, sobre todo el grupo de metas de evitación del aprendizaje (EV_AP) en lo que se refiere a la Planificación y gestión de recursos, a la que recurre rara vez. Lo cierto es que las dos agrupaciones de aproximación optan por estrategias activas para solucionar las demandas académicas, lo que les permite combatir eficazmente las posibles sensaciones de falta de control y de impotencia personal derivadas de las situaciones de estrés, destacándose como las agrupaciones de estudiantes mejor adaptadas al entorno universitario. 
En general, los resultados obtenidos en nuestro estudio nos indican que los tres grupos con motivaciones negativas de evitación del rendimiento (EV_RD) y de evitación del aprendizaje (EV_AP) o que combinan ambos tipos de metas (EV_AP+EV_RD) son las agrupaciones de estudiantes que evidencian una tendencia a la inhibición de actividad constructiva, al eludir el uso de formas de afrontamiento cognitivo y activo para hacer frente a sus dificultades académicas, lo cual no deja de ser preocupante si tenemos en cuenta que representan el 57\% de la muestra estudiada.

Sin lugar a dudas, este último dato nos sitúa ante un problema de gran calado en la universidad actual y que tiene importantes repercusiones sobre el estado de bienestar de los estudiantes. Deberíamos tener presente que una institución universitaria tiene la responsabilidad de evitar que el estrés académico se convierta en algo insano para sus miembros, de tal manera que acabe infravalorando el esfuerzo y colaborando en un mantenimiento cronificado de la tensión en el estudiante. Mejorar el entorno y controlar los estímulos negativos, los desajustes, las deficiencias del proceso de enseñanza-aprendizaje o de la planificación u organización de los estudios es una obligación de cualquier organismo con un proyecto formativo de alcance. Pero hoy en día, además, sí es prioritario que como institución mantenga su propio compromiso con la capacitación adecuada de los futuros profesionales a la que aspira y, para ello, debe asumir la necesidad de implementar programas para sus jóvenes discentes que les prepare para hacer frente de forma constructiva al estrés generado por las exigencias académicas y les ofrezca herramientas cognitivas con las que encarar en el futuro los inevitables problemas vinculados a la complejidad y, más que probable, inestabilidad de su futura vida laboral. Manejar el estrés, fomentar los recursos de afrontamiento, promover el apoyo motivacional y la afectividad positiva, formar habilidades para saber responder a las demandas (Brougham, Zail, Mendoza, y Miller, 2009; González y Landero, 2008) parecen propuestas obligadas por parte de las instituciones universitarias para ayudar al estudiante a encarar con efectividad el estrés, evitar en la medida de lo posible su vulnerabilidad frente al mismo, y acrecentar sus opciones de adaptación y su desempeño académico.

\section{REFERENCIAS}

Bisquerra, R. (1989). Introducción conceptual al análisis multivariable. Un enfoque informático con los paquetes estadísticos SPSS-X. LISREL y SPAD. Barcelona: Promociones Publicaciones Universitarias.

Brougham, R., Zail, C., Mendoza, C., y Miller, J. (2009). Stress, sex differences, and coping strategies among college students. Current Psychology, 28, 85-97. doi:10.1007/s12144009-9047-0

Cabanach, R.G., Fariña, F., Freire, C., González, P., y Ferradás, M. (2013). Diferencias en el afrontamiento del estrés en estudiantes universitarios hombres y mujeres. European Journal of Education and Psychology, 6(1), 19-32. 
Cabanach, R.G., Valle, A., Rodríguez, S., Piñeiro, I., y Freire, C. (2010). Escala de afrontamiento del estrés académico (A-CEA). Revista Iberoamericana de Psicología y Salud, 1(1), 5164.

Cabanach, R.G., Valle, A., Rodríguez, S., y Piñeiro, I. (2008). Afrontamiento del estrés en contextos universitarios: Construcción de una escala de medida. Comunicación. $V$ Congreso Internacional de Psicología y Educación: los Retos de Futuro. Oviedo. 23-25 de abril.

Fernández, M.D., y Díaz, M.A. (2001). Relación entre estrategias de afrontamiento, síndromes clínicos y trastornos de personalidad en pacientes esquizofrénicos crónicos. Revista de Psicopatología y Psicología Clínica, 6, 129-136.

Fernández-Abascal, E.G. (1999). El estrés: Aspectos básicos y de intervención. En E.G. Fernández-Abascal y F. Palmero (Eds), Emociones y salud (pp. 327-348). Barcelona: Ariel, S.A.

González, M.T., y Landero, R. (2008). Síntomas psicosomáticos y estrés: Comparación de un modelo estructural entre hombres y mujeres. Ciencia UANL, 11(4), 403-410.

IBM Corp (2013). IBM SPSS Statistics for Windows, Version 22.0. Armonk, NY: International Business Machines Corporation.

Lazarus, R.S., y Folkman, S. (1986). Estrés y procesos cognitivos. Barcelona: Martínez Roca.

Richardson, C., y Poole, H. (2001). Chronic pain and coping: a proposed role for nurses and nursing models. Journal of Advanced Nursing, 34, 659-667.

Skaalvik, E.M. (1997). Self- enhancing and self- defeating ego orientation: Relations with task and avoidance orientation, achievement, self- perceptions, and anxiety. Journal of Educational Psychology, 89, 71- 81.

Skinner, E.A., Edge, K., Altman, J., y Sherwood, H. (2003). Searching for the structure of coping: A review and critique of category systems for classifying ways of coping. Psychological Bulletin, 129, 216-269. 